\title{
Implications of the Fractional Charge of Hydroxide at the Electrochemical Interface
}

\author{
Joseph A. Gauthier, ${ }^{\dagger, \ddagger}$ Leanne D. Chen, ${ }^{\dagger, \S}$ Michal Bajdich, ${ }^{\ddagger}$ and Karen Chan*, ${ }^{*}$ \\ $\dagger$ †UNCAT Center for Interface Science and Catalysis, Department of Chemical \\ Engineering, Stanford University, Stanford, California 94305, United States \\ $\ddagger S U N C A T$ Center for Interface Science and Catalysis, SLAC National Accelerator \\ Laboratory, 2575 Sand Hill Road, Menlo Park, California 94025, United States \\ \Department of Physics, Technical University of Denmark, DK-2800, Kgs. Lyngby, \\ Denmark \\ $\S$ Present Address: Division of Chemistry and Chemical Engineering, California Institute of \\ Technology, Pasadena, California 91125, United States \\ E-mail: kchan@fysik.dtu.dk
}




\begin{abstract}
Rational design of materials that efficiently convert electrical energy into chemical bonds will ultimately depend on a thorough understanding of the electrochemical interface at the atomic level. Towards this goal, the use of density functional theory (DFT) at the generalized gradient approximation (GGA) level has been applied widely in the past 15 years. In the calculation of electrochemical reaction energetics using GGA-DFT, it is frequently implicitly assumed that ions in the Helmholtz plane have unit charge. However, the ion charge is observed to be fractional near the interface through both a capacitor model and through Bader charge partitioning. In this work, we show that this spurious charge transfer can be effectively mitigated by continuum charging of the electrolyte. We then show that, similar to hydronium, the observed fractional charge of hydroxide is not due to a GGA level self-interaction error, as the partial charge is observed even when using hybrid level exchange-correlation functionals.
\end{abstract}

\title{
Graphical TOC Entry
}

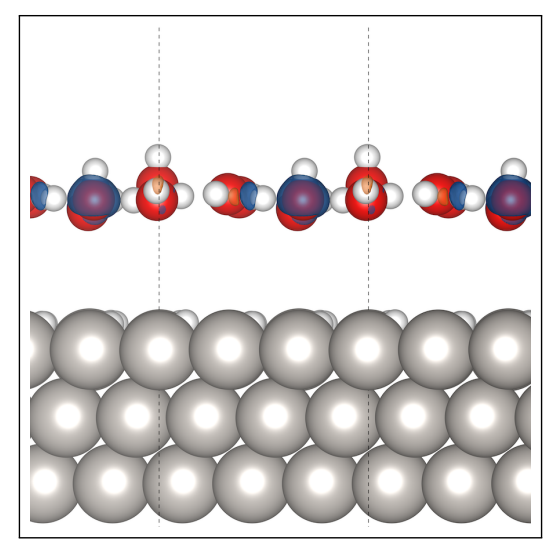




\section{Introduction}

The electrochemical conversion of chemicals into value added-commodity products has been a growing interest to the scientific community in the past several decades, owing largely to the rapidly decreasing cost of renewable electricity from solar photovoltaics. ${ }^{1,2}$ The rapid decrease in the cost of electricity allows processes such as the reduction of $\mathrm{CO}_{2}$ and $\mathrm{N}_{2}$ closer to be increasingly commercially viable despite significant limitations by poor catalytic activities of current catalysts ${ }^{3-8}$ In particular, $\mathrm{CO}_{2}$ and $\mathrm{N}_{2}$ reduction catalysts are both limited by activity and selectivity. Ultimately, the rational design of catalysts for these and other related processes will rely on a detailed, atomic-scale understanding of the electrochemical interface. ${ }^{9-12}$ Computational efforts in this space are dominated by density functional theory (DFT) methods at the generalized gradient approximation (GGA) level, since these offer a good balance between cost and accuracy. ${ }^{13-15}$ However, GGA-DFT methods have long been known to poorly predict the band gaps of semiconducting materials, ${ }^{16}$ barriers of chemical reactions, energies of dissociating molecular ions, and charge transfer excitation energies all due to a well-know delocalization error. ${ }^{17-20}$ The same challenges with band gap prediction can lead to spurious charge transfer between the electrolyte and metal slab in electrochemical systems if the electrolyte band gap does not properly straddle the metal Fermi level, commonly referred to as spurious charge transfer (SCT). ${ }^{17,21}$

In a recent study, ${ }^{22}$ we showed using higher level hybrid DFT methods that the observed partial charge on hydronium is not caused by these delocalization errors in GGA-DFT. Rather, it is caused by a physical electron charge density overlap between the ion and the nearby metal surface. The extension of this argument to the case of hydroxide is technically complicated by the aforementioned issues of SCT, where the highest occupied molecular orbital (HOMO) of the hydroxide ion often aligns with the metal Fermi level for metals with a relatively large work function such as Pt. In this work, we show that the challenge of SCT can be effectively mitigated by the placement of an excess surface charge density, with countercharges described by, e.g., the linearized Poisson-Boltzmann equation. When the 
electrolyte bands properly straddle the metal Fermi level, i.e. the Fermi level lies within the electrolyte bandgap without any alignment with either the HOMO or the lowest unoccupied molecular orbital (LUMO) bands, we show that the partial charge observed on hydroxide is also not caused by delocalization errors. The fractional charge of hydroxide has implications for kinetic studies of alkaline reactions, where hydroxide at the interface plays a critical role.

\section{Theoretical methods and models}

This work uses the Vienna ab-initio Software Package ${ }^{23-25}$ in conjunction with the added functionality of implicit solvation provided by VASPsol. ${ }^{26,27}$ Core electrons are described as projector augmented wave pseudopotentials, ${ }^{28}$ while valence electrons are expanded as planewaves up to a kinetic energy cutoff of $400 \mathrm{eV}$. Electron exchange and correlation interactions are accounted for using the PBE functional ${ }^{29}$ at the GGA level and with the HSE06 ${ }^{30}$ functional for hybrid-level calculations. When optimizing bulk platinum to determine the appropriate lattice constant, the Brillouin zone is sampled with a $12 \times 12 \times 12$-centered Monkhorst-Pack ${ }^{31} k$-point mesh. The optimized lattice constant is determined to be 3.968 $\AA$ for PBE functional, which is very close to exprimental value of $3.912 \AA$ ?

A $3 \times 3$ supercell of $\mathrm{Pt}$ (111) surface with a single bilayer of water is used as the model system. A slab thickness of 3 layers, with the bottom two layers being fixed at the bulk lattice constant value, is used to describe the electrode. A complete monolayer of hydrogen is placed on the surface to facilitate stabilization of the hydroxide ion. The geometries are optimized in the case of a hydronium and hydroxide in the water layer, separately. The forces are minimized until the maximum force on all unconstrained atoms is less than $0.03 \mathrm{eV} \AA^{-1}$. The solvent bilayer is systematically moved away from the surface to probe the effect of distance from the surface on the calculated ion charge. For these calculations, single-point energy calculations are performed to prevent water reorganization from complicating the calculated electron distribution or density of states (DOS). The computational and structural data is 
also available online at Catalysis-hub.org. ${ }^{32,33}$

VASPsol treats the electrolyte at the electrochemical interface as a polarizable continuum, placing point counter-charges via the linearized Poisson-Boltzmann equation. A Debye screening length of $3 \AA$ is chosen, which corresponds to a bulk electrolyte concentration of 1 Molar. As described in a recent work, ${ }^{34}$ the non-electrostatic coefficient is set to zero to avoid numerical instabilities in the electrolyte region. Details regarding the implementation of VASPsol can be found in its documentation. ${ }^{26,27}$

Ionic charges are determined through the Bader charge partitioning scheme, ${ }^{35-37}$ which is used to calculate the charge on each atom by subtracting the expected number of electrons on the neutral atom. Due to the ambiguity intrinsic to charge partitioning (i.e. shared electrons between covalently bonded atoms), when calculating the ionic charge, we sum the charge of every atom in the solvent layer.

\section{Results and discussion}

We begin by showing that the previously reported spurious charge transfer associated with DFT calculated band misalignment, ${ }^{16,21}$ exacerbated by solvated ions at the interface, can be mitigated through the use of continuum charging. This is similar to the homogeneously charged background approach previously used. ${ }^{21}$ By charging the surface, the HOMO can be shifted relative to the Fermi level sufficiently to avoid SCT. This charging method allows us to investigate the behavior of anions at the interface where previously SCT made this impractical. We then show that the fractional charge of ions near the charged electrochemical interface is not due to GGA level delocalization errors by comparing to hybrid-level (HSE06 ${ }^{30,38}$ ) calculations. Previous analysis of the hydronium ion rationalized the partial charge by the asymmetric overlap of electron charge density between the metal slab and the ion in solution. We show here that this analysis cannot be extended to hydroxide, but the non-unity charge is nonetheless not caused by DFT delocalization errors. 


\section{Avoiding spurious charge transfer with continuum charging}

Recently, the well-known challenge of DFT systematically under-predicting band gaps ${ }^{16}$ was

shown to occasionally cause spurious charge transfer at the metal/electrolyte interface, ${ }^{21}$ by either the HOMO or LUMO of the electrolyte not appropriately straddling the metal Fermi level. This challenge, commonly referred to as SCT, is in practice exacerbated by ions (especially anions) between the electrochemical interface. 

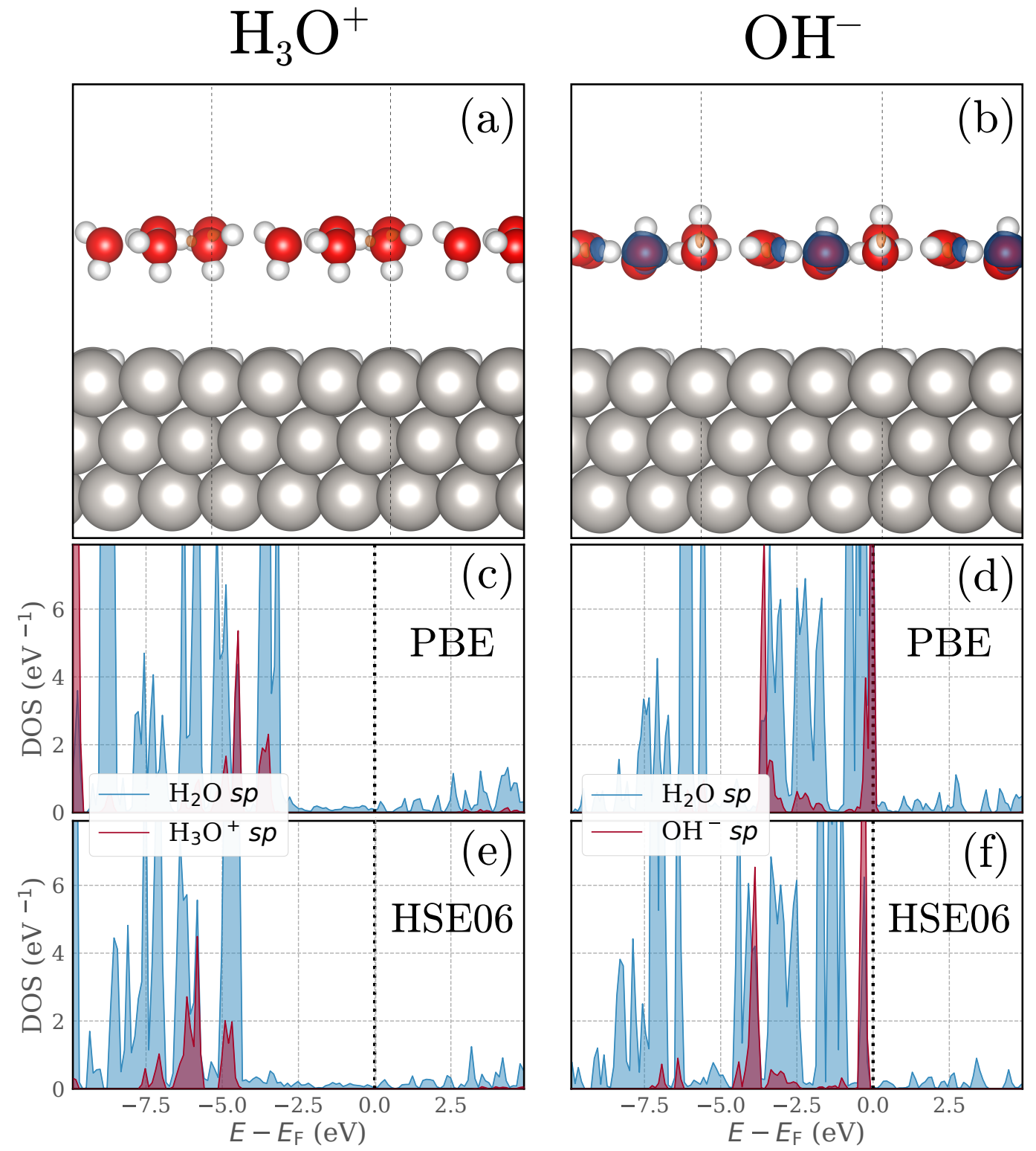

Figure 1: Charge density difference between hybrid and GGA level DFT calculations corresponding to (a) a case with a hydronium ion near the surface, not showing SCT, and (b) a case with a hydroxide ion near the surface, showing SCT. The isosurface values of $\pm 2.5 \cdot 10^{-3}$ $\mathrm{e} \AA^{-3}$ corresponds to higher electron density (orange, more negatively charged) and lower electron density (blue, more positively charged). Illustrated here is the spurious transfer of charge from the electrolyte to the metal surface, totalling about 0.2 electrons. Panels (c) and (d) show the projected density of states (pDOS) at the GGA level for hydronium and hydroxide, respectively, where the incorrect extension of the hydroxide HOMO to above the Fermi level is clearly seen in panel (d). Finally, panels (e) and (f) show the pDOS at the hybrid level for hydronium and hydroxide, respectively; with the hybrid functional, the HOMO of hydroxide is shifted to be correctly below the Fermi level. 
The lower edge of the LUMO for cations tends to be positioned below the Fermi level, while the upper edge of the HOMO for anions often extends above the Fermi level. This band misalignment results in the spurious transfer of electrons from the metal to the ion in the former case, and from ion to metal in the latter case. To illustrate this effect of GGA-DFT overdelocalizing electrons compared to hybrid-DFT, we calculate a charge density difference $\rho_{\text {diff as: }}$

$$
\rho_{\text {diff }}=\rho_{\mathrm{GGA}}-\rho_{\mathrm{HSE} 06} .
$$

Here $\rho_{\text {diff }}$ is the charge density difference, $\rho_{\mathrm{GGA}}$ is the charge density calculated at the GGA level using PBE as the exchange-correlation functional, and $\rho_{\mathrm{HSE} 06}$ is the charge density calculated using a single-point calculation on the GGA level geometry. Figure 1, panels (a) and (b), show this charge density difference for a case not showing SCT (hydronium) and a case showing SCT (hydroxide), respectively. The geometries shown correspond to the explicit solvent bilayer shifted about $2 \AA$ from the equilibrium position, where the misalignment becomes more pronounced, to more clearly demonstrate SCT.

Figure 1 (c) and (e) shows the projected density of states (pDOS) of the electrochemical interface with a hydronium ion in the outer Helmholtz plane at the GGA and hybrid level, respectively. In both cases, the electrolyte has no states at the Fermi level, indicating that the system is not exhibiting SCT. In these systems, the ionic charge was determined to be $1.0 e$ at both the GGA and hybrid level. The charge here is not fractional since the bilayer has been shifted away from the surface. This offset effectively removes the electron density overlap effect previously reported for acidic conditions. ${ }^{22}$

A similar analysis is shown for the case of a hydroxide ion in the outer Helmholtz plane in Figure 1 (d) and (f), at the GGA and hybrid levels, respectively. Here the hydroxide ion shows a clear case of SCT at the GGA level, with the hydroxide HOMO states spilling over the metal Fermi level. This results in spurious charge transfer from the electrolyte to the metal, causing the predicted ionic charge to differ: a charge of $0.81 e$ at the GGA level, and a charge of $1.0 e$ at the hybrid level. 
Similar to what was shown in Ref. 21, SCT can be mitigated by increasing or decreasing the electron density on the metal surface. We demonstrate this here by charging the surface, with the placement of counter-charges determined by solving the linearized PoissonBoltzmann equation as implemented in VASPsol, thereby retaining overall charge neutrality of the system. In Figure 2, we plot the pDOS of the water and hydroxide ion at the GGA level for three different surface charges $\sigma$, and at the hybrid level in a simulation without excess charging of the surface. As the surface charge density is systematically increased, the hydroxide HOMO is shifted negatively, until there is no spillover to above the Fermi level at a charge of $\sigma=0.75$ excess electrons, i.e. the integral of hydroxide pDOS states above the Fermi level is equal to zero. 

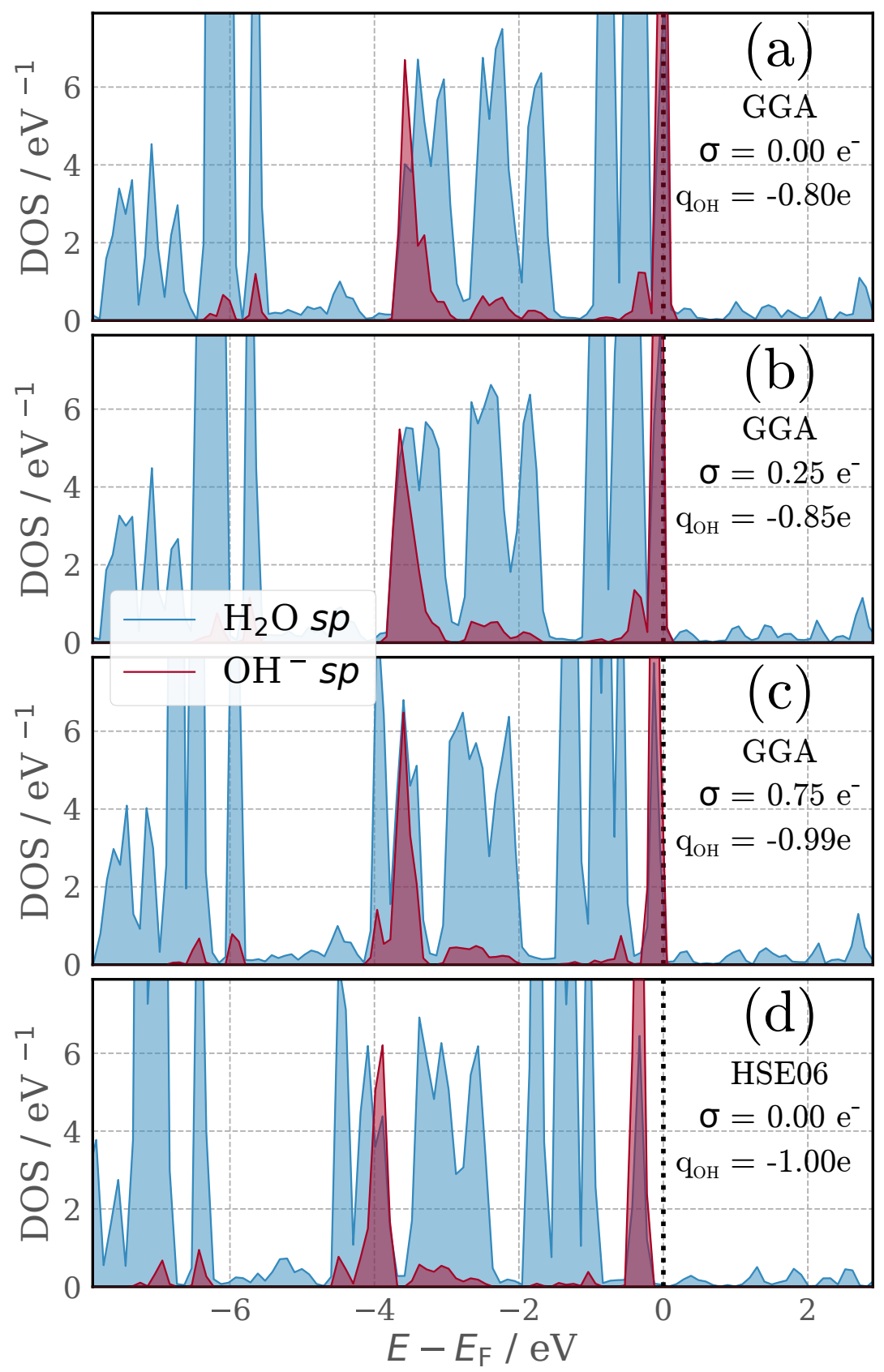

Figure 2: Illustration of the mitigation of SCT by continuum charging. By increasing the surface charge density, the HOMO energies of both the ion and the solvent are lowered relative to the metal Fermi level. Panels (a), (b), and (c) show the pDOS of the electrolyte at the GGA level for an increasing surface charge density. Panel (d) shows the pDOS at the hybrid level. 


\section{Implications of the fractional charge of hydroxide}

With the ability to mitigate SCT by charging the surface established, we now turn our attention to rationalizing the observed fractional charge of the hydroxide ion near the metal surface. We follow a similar approach to our previous work, ${ }^{22}$ and measure the charge on different parts of the system as the ion is systematically moved from its equilibrium position at the interface. Single point calculations are performed at each position to prevent water structure reorientation from conflating the resulting charge measurement, illustrated in Figure 3.

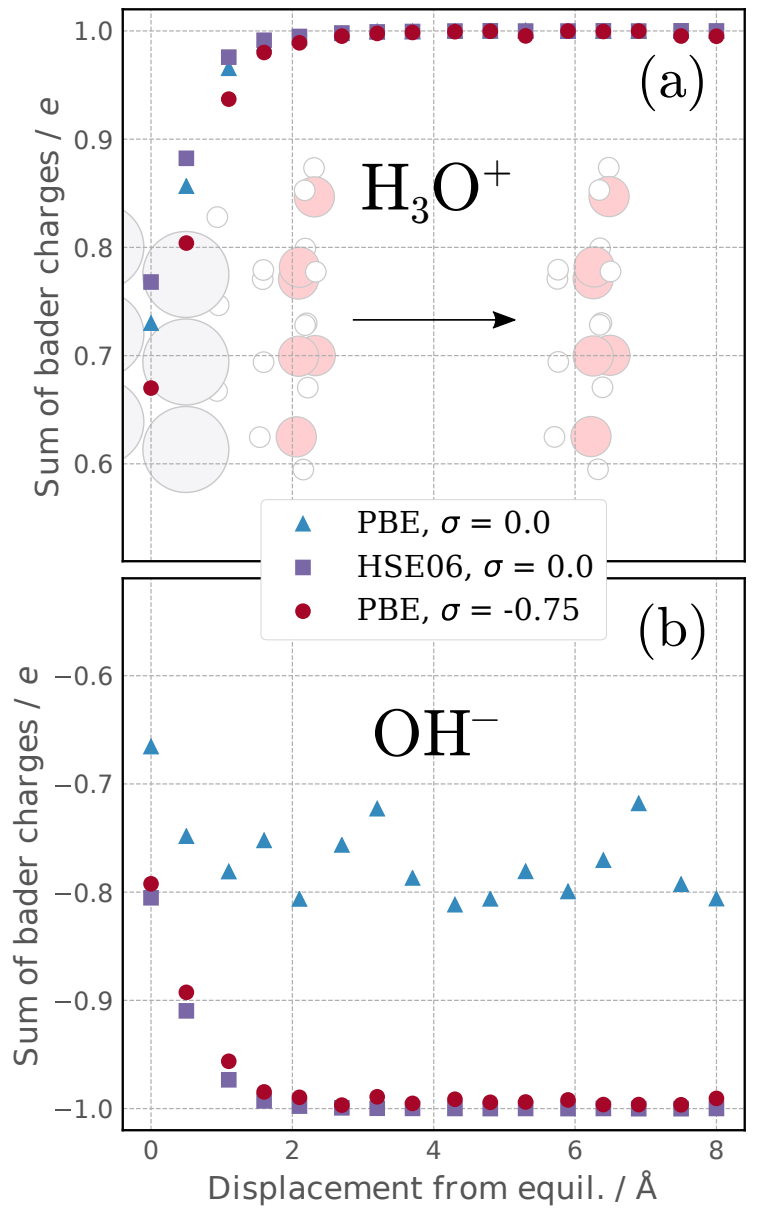

Figure 3: Ionic charge, determined by summing the Bader charge of all electrolyte molecules, as a function of the distance from equilibrium near the metal surface, for (a) hydronium, and (b) hydroxide. For the GGA level with no added continuum charge (i.e. PBE, $\sigma=0.0$ ), hydroxide exhibits clear SCT, with the ion charge being partial at all distances from the surface. 
The ions are stabilized far from the surface with continuum solvation through VASPsol. To determine the charge on the ion at a given distance from equilibrium, the Bader charge partitioning scheme ${ }^{35-37}$ is used to determine the charge on each atom in the simulation. Following the approach used previously, ${ }^{22}$ the total ionic charge $q_{\text {ion }}$ is given by

$$
q_{\text {ion }}=\sum_{i}^{n_{\text {solv. }}} q_{i}
$$

Here $n_{\text {solv. }}$ is the number of atoms in the electrolyte, and $q_{i}$ is the Bader charge on the given atom in the electrolyte. In other words, the ionic charge is taken to be the sum of all charges on atoms in the solvent bilayer. We show the above ionic charge $q_{\text {ion }}$ for both hydronium and hydroxide as a function of its position relative to equilibrium in Figure 3, at both the GGA and hybrid level with varying amounts of the total number of electrons in the system.

Figure 3(a) shows the hydronium ionic charge as the solvent bilayer is moved away from the surface at both the GGA and hybrid levels. This analysis was carried out both with and without additional continuum charging. As we reported previously, the agreement between the GGA and hybrid level is quite good, ${ }^{22}$ with less than $0.05 e$ difference at all points. The addition of excess surface electron density on the surface changes the measured ionic charge near the surface. Excess surface charge changes the electron density decay behavior of the surface, thereby affecting the resulting ionic charge by about $0.06 e$ in the case of hydronium when 0.75 excess electrons are put into the simulation.

Unlike the case of hydronium, the partial charge on hydroxide is not easily rationalized from a charge density overlap analysis, as we show in Supplementary Info Note 1. Furthermore, a direct comparison of the GGA and hybrid level is complicated by SCT with no excess surface charge, shown in Figure 3(b). As the hydroxide ion is moved away from the surface with $\sigma=0.0$, the calculated ionic charge exhibits instability, fluctuating between 0.7 and $0.8 e$, largely due to SCT. When excess surface charge is introduced, pushing the system out of SCT, the measured ionic charge stabilizes and agrees quite well with the hybrid results. 
We note that, as seen in the case of hydronium, Figure 3 (a), the excess surface charge can affect the calculated ionic charge near the surface. Since this perturbation resulted in less than $0.1 e$ change in ionic charge in the case of hydronium, we conclude that the hybrid and GGA levels agree in the case of hydroxide.

The partial ion charge has implications for the potential dependence of reactions involving the ion; for example, coupled proton-electron transfer reactions which can utilize both hydronium and hydroxide as a donor. As we outlined in recent works, ${ }^{39,40}$ the potential dependence of a chemical reaction is given by the slope of the reaction energy as a function of the work function averaged between the initial and final states. In the case of the Volmer reaction in acid (i.e. $\left(\mathrm{H}^{+}+\mathrm{e}^{-}\right)+* \rightarrow \mathrm{H} *$ ), this slope is approximately $0.7,{ }^{40-43}$ corresponding to the partial charge of the ion near the surface. ${ }^{22}$ Shown in Figure 4 is the Volmer reaction energy in base (i.e. $\mathrm{H}_{2} \mathrm{O}+\mathrm{e}^{-}+{ }^{*} \rightarrow \mathrm{H}^{*}+\mathrm{OH}^{-}$), showing a potential dependence of approximately 0.7 . In other words, changing the potential of the electrode by $1 \mathrm{~V}$ only changes the reaction energy by $0.7 \mathrm{eV}$. The remaining potential dependence must then be accounted for by the reaction process (in the case of acidic Volmer) bringing a proton from the bulk to a position close to the surface, or (in the case of alkaline Volmer) moving the hydroxide from the surface to the bulk. 


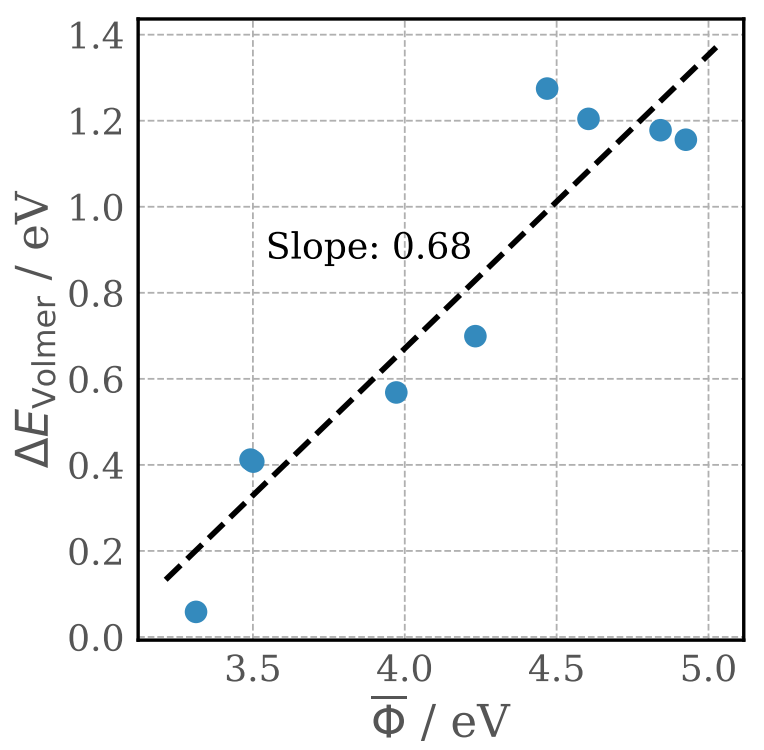

Figure 4: Alkaline Volmer reaction energy, i.e. $\mathrm{H}_{2} \mathrm{O}+\mathrm{e}^{-}+{ }^{*} \rightarrow \mathrm{H}^{*}+\mathrm{OH}^{-}$, as a function of potential showing a slope of 0.7 . This potential dependence is the same as that observed in the acidic Volmer reaction, i.e. $\left(\mathrm{H}^{+}+\mathrm{e}^{-}\right)+* \rightarrow \mathrm{H} *$.

\section{Conclusions}

To summarize, we have shown here that due to the well-known failure of DFT to accurately describe band levels, the treatment of ions at the interface with GGA level DFT can be technically challenging, especially in the case of anions. However, these issues can be mitigated by introducing excess surface charge, with countercharge placed by (for instance) solving the linearized Poisson-Boltzmann equation as implemented in the VASPsol. We then show that hydroxide ions in the outer Helmholtz plane have a fractional charge of -0.8 to -0.7 $e$ at both the GGA and hybrid levels of DFT. Although this fractional charge cannot easily be explained by a simple spillover analysis as in the case of hydronium, we show that the fractional charge is not a result of the DFT delocalization error, since both the GGA and hybrid levels predict a charge of unity as the ion moves away from the interface. This work has implications for electrochemical kinetics involving reactions in basic media, since the calculation of electrochemical barriers typically involves either the initial or final state with an ion in the outer Helmholtz plane. Whereas many previous works using the computational 
hydrogen electrode $(\mathrm{CHE})^{44}$ assume an equilibrium between the ion in the Helmholtz plane and the ion in bulk, we show here that this need not be the case.

\section{Acknowledgement}

This work was performed under the Joint Center for Artificial Photosynthesis, a DOE Energy Innovation Hub, supported through the Office of Science of the U.S. Department of Energy under Award Number DE-SC0004993. This work uses computational resources at the the Stanford Research Computing Center and also of the National Energy Research Scientific Computing Center, a DOE Office of Science User Facility, supported by the Office of Science

of the U.S. Department of Energy under Contract No. DE-AC02-05CH11231. Some of the computing for this project was performed on the Sherlock cluster. KC acknowledges support by a research grant (9455) from VILLUM FONDEN

\section{References}

(1) Seh, Z. W.; Kibsgaard, J.; Dickens, C. F.; Chorkendorff, I.; Nørskov, J. K.; Jaramillo, T. F. Combining theory and experiment in electrocatalysis: Insights into materials design. Science 2017, 355.

(2) Lazard's Levelized Cost of Energy Analysis - Version 12.0. 2018; https : //www . lazard. com/media/450784/lazards-levelized-cost-of-energy-version-120-vfinal. pdf.

(3) De Luna, P.; Hahn, C.; Higgins, D.; Jaffer, S. A.; Jaramillo, T. F.; Sargent, E. H. What would it take for renewably powered electrosynthesis to displace petrochemical processes? Science 2019, 364, eaav3506.

(4) Jouny, M.; Luc, W.; Jiao, F. General techno-economic analysis of CO2 electrolysis systems. Industrial \& Engineering Chemistry Research 2018, 57, 2165-2177. 
(5) Singh, A. R.; Rohr, B. A.; Schwalbe, J. A.; Cargnello, M.; Chan, K.; Jaramillo, T. F.; Chorkendorff, I.; Nørskov, J. K. Electrochemical Ammonia Synthesis: The Selectivity Challenge. 2016,

(6) McEnaney, J. M.; Singh, A. R.; Schwalbe, J. A.; Kibsgaard, J.; Lin, J. C.; Cargnello, M.; Jaramillo, T. F.; Nørskov, J. K. Ammonia synthesis from N 2 and H 2 O using a lithium cycling electrification strategy at atmospheric pressure. Energy $\&$ Environmental Science 2017, 10, 1621-1630.

(7) Wang, L.; Xia, M.; Wang, H.; Huang, K.; Qian, C.; Maravelias, C. T.; Ozin, G. A. Greening ammonia toward the solar ammonia refinery. Joule 2018, 2, 1055-1074.

(8) Nitopi, S.; Bertheussen, E.; Scott, S. B.; Liu, X.; Engstfeld, A. K.; Horch, S.; Seger, B.; Stephens, I. E.; Chan, K.; Hahn, C. et al. Progress and Perspectives of Electrochemical CO2 Reduction on Copper in Aqueous Electrolyte. Chemical reviews 2019,

(9) Creazzo, F.; Galimberti, D. R.; Pezzotti, S.; Gaigeot, M.-P. DFT-MD of the (110)Co3O4 cobalt oxide semiconductor in contact with liquid water, preliminary chemical and physical insights into the electrochemical environment. The Journal of chemical physics 2019, 150, 041721.

(10) Klimeš, J.; Bowler, D. R.; Michaelides, A. Chemical accuracy for the van der Waals density functional. Journal of Physics: Condensed Matter 2009, 22, 022201.

(11) Carrasco, J.; Hodgson, A.; Michaelides, A. A molecular perspective of water at metal interfaces. Nature materials 2012, 11, 667.

(12) Gray, C. M.; Saravanan, K.; Wang, G.; Keith, J. A. Quantifying solvation energies at solid/liquid interfaces using continuum solvation methods. Molecular Simulation 2017, 43, 420-427. 
(13) Wellendorff, J.; Lundgaard, K. T.; Møgelhøj, A.; Petzold, V.; Landis, D. D.; Nørskov, J. K.; Bligaard, T.; Jacobsen, K. W. Density functionals for surface science: Exchange-correlation model development with Bayesian error estimation. Physical Review B 2012, 85, 235149 .

(14) Tran, F.; Stelzl, J.; Blaha, P. Rungs 1 to 4 of DFT JacobâĂŹs ladder: extensive test on the lattice constant, bulk modulus, and cohesive energy of solids. The Journal of chemical physics 2016, 144, 204120.

(15) Perdew, J. P.; Schmidt, K. Jacob's Ladder of Density Functional Approximations for the Exchange-Correlation Energy. AIP Conference Proceedings 2001, 577, 1-20.

(16) Perdew, J. P. Density functional theory and the band gap problem. International Journal of Quantum Chemistry 1985, 28, 497-523.

(17) Cohen, A. J.; Mori-Sánchez, P.; Yang, W. Insights into current limitations of density functional theory. Science 2008, 321, 792-794.

(18) Lundberg, M.; Siegbahn, P. E. Quantifying the effects of the self-interaction error in DFT: When do the delocalized states appear? The Journal of chemical physics 2005, 122, 224103.

(19) Mori-Sánchez, P.; Cohen, A. J.; Yang, W. Localization and delocalization errors in density functional theory and implications for band-gap prediction. Physical review letters 2008, 100, 146401.

(20) Li, C.; Zheng, X.; Cohen, A. J.; Mori-Sánchez, P.; Yang, W. Local scaling correction for reducing delocalization error in density functional approximations. Physical review letters 2015, 114, 053001.

(21) Björketun, M. E.; Zeng, Z.; Ahmed, R.; Tripkovic, V.; Thygesen, K. S.; Rossmeisl, J. 
Avoiding pitfalls in the modeling of electrochemical interfaces. Chemical Physics Letters 2013, 555, 145-148.

(22) Chen, L. D.; Bajdich, M.; Martirez, J. M. P.; Krauter, C. M.; Gauthier, J. A.; Carter, E. A.; Luntz, A. C.; Chan, K.; Nørskov, J. K. Understanding the apparent fractional charge of protons in the aqueous electrochemical double layer. Nature Communications 2018, 9, 3202.

(23) Kresse, G.; Hafner, J. Ab initio molecular dynamics for liquid metals. Physical Review $B$ 1993, 47, 558-561.

(24) Kresse, G.; Furthmüller, J. Efficient iterative schemes for ab initio total-energy calculations using a plane-wave basis set. Physical Review B 1996, 54, 11169-11186.

(25) Kresse, G.; Furthmüller, J. Efficiency of ab-initio total energy calculations for metals and semiconductors using a plane-wave basis set. Computational Materials Science 1996, $6,15-50$.

(26) Mathew, K.; Hennig, R. G. Implicit self-consistent description of electrolyte in planewave density-functional theory. arXiv preprint arXiv:1601.03346 2016,

(27) Mathew, K.; Sundararaman, R.; Letchworth-Weaver, K.; Arias, T.; Hennig, R. G. Implicit solvation model for density-functional study of nanocrystal surfaces and reaction pathways. The Journal of Chemical Physics 2014, 140, 084106.

(28) Kresse, G. From ultrasoft pseudopotentials to the projector augmented-wave method. Physical Review B 1999, 59, 1758-1775.

(29) Perdew, J. P.; Burke, K.; Ernzerhof, M. Generalized Gradient Approximation Made Simple. Physical Review Letters 1996, 77, 3865-3868.

(30) Krukau, A. V.; Vydrov, O. A.; Izmaylov, A. F.; Scuseria, G. E. Influence of the exchange 
screening parameter on the performance of screened hybrid functionals. The Journal of chemical physics 2006, 125, 224106.

(31) Monkhorst, H. J.; Pack, J. D. Special points for Brillouin-zone integrations. Physical Review B 1976, 13, 5188.

(32) Winther, K. T.; Hoffmann, M. J.; Boes, J. R.; Mamun, O.; Bajdich, M.; Bligaard, T. Catalysis-Hub. org, an open electronic structure database for surface reactions. Scientific data 2019, 6, 75 .

(33) Gauthier, J. A.; Chen, L. D.; Bajdich, M.; Chan, K. https://www. catalysis-hub. org/publications/GauthierImplications2019.

(34) Gauthier, J. A.; Ringe, S.; Dickens, C. F.; Garza, A. J.; Bell, A. T.; Head-Gordon, M.; Nørskov, J. K.; Chan, K. Challenges in Modeling Electrochemical Reaction Energetics with Polarizable Continuum Models. ACS Catalysis 2018, 9, 920-931.

(35) Tang, W.; Sanville, E.; Henkelman, G. A grid-based Bader analysis algorithm without lattice bias. Journal of Physics: Condensed Matter 2009, 21, 084204.

(36) Sanville, E.; Kenny, S. D.; Smith, R.; Henkelman, G. Improved grid-based algorithm for Bader charge allocation. Journal of Computational Chemistry 2007, 28, 899-908.

(37) Henkelman, G.; Arnaldsson, A.; Jónsson, H. A fast and robust algorithm for Bader decomposition of charge density. Computational Materials Science 2006, 36, 354-360.

(38) Heyd, J.; Scuseria, G. E.; Ernzerhof, M. Hybrid functionals based on a screened Coulomb potential. The Journal of chemical physics 2003, 118, 8207-8215.

(39) Gauthier, J.; Dickens, C.; Ringe, S.; Chan, K. Practical Considerations for Continuum Models Applied to Surface Electrochemistry. 2019,

(40) Gauthier, J.; Dickens, C.; Heenen, H. H.; Ringe, S.; Chan, K. Unified approach to implicit and explicit solvent simulations of electrochemical reaction energetics. 2019, 
(41) Chan, K.; Nørskov, J. K. Potential dependence of electrochemical barriers from ab initio calculations. The Journal of Physical Chemistry Letters 2016, 7, 1686-1690.

(42) Chan, K.; Nørskov, J. K. Electrochemical barriers made simple. The Journal of Physical Chemistry Letters 2015, 6, 2663-2668.

(43) Sakong, S.; Groß, A. The electric double layer at metal-water interfaces revisited based on a charge polarization scheme. The Journal of Chemical Physics 2018, 149, 084705.

(44) Nørskov, J. K.; Rossmeisl, J.; Logadottir, A.; Lindqvist, L.; Kitchin, J. R.; Bligaard, T.; Jonsson, H. Origin of the overpotential for oxygen reduction at a fuel-cell cathode. The Journal of Physical Chemistry B 2004, 108, 17886-17892. 